stant for the solute, and $\mathrm{K}_{\mathrm{c}}$ and $\mathrm{K}_{\mathrm{d}}$ hindrance factors for convection and diffusion, respectively.

Measurements on isolated glomerular basement membranes ${ }^{2}$ suggest that $\Phi \mathrm{K}_{\mathrm{c}}=0.2$ and $\Phi K_{d}=0.02$. The latter value shows that diffusion is markedly impaired in the glomerular basement membrane as compared with diffusion in water ( $2 \%$ of $\mathrm{D}$ ), but convective transport is also restricted. The key point is whether diffusion is more limited than convection; the Pe reflects this. A Pe above unity indicates that transport occurs mainly by convection, and a Pe of less than 1 reflects diffusion-dominated transport.

The Pe in glomerular basement membrane for VEGF can be estimated by using a value of $v$ close to $4 \times 10^{-4} \mathrm{~cm}$ per second and $\delta$ close to $2 \times 10^{-5} \mathrm{~cm}$. VEGF has a Stokes-Einstein radius $\left(\mathrm{r}_{\mathrm{s}}\right)$ of $2.6 \mathrm{~nm}$, which implies that $\mathrm{D}_{2.6 \mathrm{~nm}}$ is $1.26 \times 10^{-6} \mathrm{~cm}^{2}$ per second. Inserting these values into equation 1 results in a Pe of 0.063 . It is safe to conclude that VEGF is transported by diffusion, and diffusion alone, across the glomerular basement membrane.

The model presented by Katavetin and Katavetin has the virtue of simplicity but would appear to be an oversimplification. For example, one needs to incorporate the effects of serial barriers ${ }^{1}$ to allow for better predictions of concentrations at the endothelium.

Also, as stated by Katavetin and Katavetin, the general perception is that transport across the glomerular barrier is completely dominated by convection. In fact, the opposite is true. ${ }^{2}$ Thus, the flow conditions in the glomerular basement membrane resemble not a waterfall but, rather, a great lake with slow flow velocity. Consequently, diffusion dominates the transport of most solutes in the glomerular basement membrane. ${ }^{2}$
We thank Nochy et al. for pointing out an additional published case report by Frangié et al. ${ }^{3}$ The issue of reversibility of thrombotic microangiopathy raised by the authors is important. ${ }^{4} \mathrm{Al}-$ though renal function improved in the six cases we reported, we were unable to determine whether it returned to baseline. Frangié et al. found that although hypertension and hemolysis improved, the proteinuria persisted between cycles; this may reflect podocyte loss and segmental sclerosis, as shown in their report. We are aware of a patient at the University of Toronto in whom irreversible chronic kidney injury apparently developed and progressed to end-stage renal failure, despite discontinuation of the VEGF inhibitor.

Borje Haraldsson, M.D., Ph.D.

Sahlgrenska University Hospital

SE-413 45 Gothenburg, Sweden

Laura Barisoni, M.D.

New York University School of Medicine

New York, NY 10016

Susan E. Quaggin, M.D.

University of Toronto

Toronto, ON M5S 1A8, Canada

quaggin@mshri.on.ca

1. Haraldsson B, Nyström J, Deen WM. Properties of the glomerular barrier and mechanisms of proteinuria. Physiol Rev 2008; 88:451-87.

2. Deen WM, Lazzara MJ, Myers BD. Structural determinants of glomerular permeability. Am J Physiol Renal Physiol 2001; 281:F579-F596.

3. Frangié $\mathrm{C}$, Lefaucheur C, Medioni J, Jacquot C, Hill GS, Nochy D. Renal thrombotic microangiopathy caused by anti-VEGFantibody treatment for metastatic renal-cell carcinoma. Lancet Oncol 2007;8:177-8.

4. Patel TV, Morgan JA, Demetri GD, et al. A preeclampsia-like syndrome characterized by reversible hypertension and proteinuria induced by the multitargeted kinase inhibitors sunitinib and sorafenib. J Natl Cancer Inst 2008;100:282-4.

\title{
Case 11-2008: Mental-Status Changes after Liver Transplantation
}

TO THE EDITOR: We would like to make two points about the case of disseminated cryptococcosis in a liver-transplant recipient, discussed in the Case Records by Fishman et al. (April 10 issue). ${ }^{1}$ The first point concerns delayed diagnosis of this infection in patients with negative tests for human immunodeficiency virus infection. We believe that in the case presented, a correct diagno- sis could have been suspected from day 13 onward on the basis of the patient's recurrent headaches. In immunocompromised patients, severe and persistent headache should prompt the performance of lumbar puncture. ${ }^{2}$

The second point is the low awareness among physicians of the diagnostic utility of cryptococcal antigen determination in serum. ${ }^{3}$ As shown 


\begin{tabular}{|c|c|c|c|c|c|}
\hline Study & $\begin{array}{l}\text { No. of } \\
\text { Patients }\end{array}$ & $\begin{array}{c}\text { Serum Cryptococcal } \\
\text { Antigen }\end{array}$ & CSF Antigen & CSF Culture & India Ink \\
\hline & & \multicolumn{4}{|c|}{ no. with positive result/total no.(\%) } \\
\hline Husain et al. ${ }^{4}$ & 125 & $18 / 21(86)$ & $37 / 37(100)$ & $76 / 82(93)$ & $36 / 47(77)$ \\
\hline Wu et al..$^{5}$ & 28 & $20 / 22(91)$ & $28 / 28(100)$ & $21 / 28(75)$ & $14 / 28(50)$ \\
\hline Total & 153 & $38 / 43(88)$ & $65 / 65(100)$ & $97 / 110(88)$ & $50 / 75(67)$ \\
\hline
\end{tabular}

* CSF denotes cerebrospinal fluid.

in Table 1, of 153 organ-transplant recipients with cryptococcal meningitis in two reports, only 43 (28\%) underwent serum cryptococcal antigen testing. ${ }^{4,5}$ This is surprising, since in transplant recipients and patients with AIDS, determination of serum cryptococcal antigen is highly sensitive and only slightly less specific than cryptococcal antigen determination in the cerebrospinal fluid. We thus advocate assessment of serum cryptococcal antigen in all immunocompromised patients with fever or headache lasting more than 3 days, whether or not meningeal signs are present.

Spinello Antinori, M.D.

Mario Corbellino, M.D.

University of Milan

20122 Milan, Italy

spinello.antinori@unimi.it

1. Case Records of the Massachussets General Hospital (Case 11-2008). N Engl J Med 2008;358:1604-13.

2. Antinori S. Signs of meningeal irritation: what is their diagnostic accuracy? Clin Infect Dis 2003;36:125-6.

3. Antinori S, Radice A, Galimberti L, Magni C, Fasan M, Parravicini $\mathrm{C}$. The role of cryptococcal antigen assay in diagnosis and monitoring of cryptococcal meningitis. J Clin Microbiol 2005;43:5828-9.

4. Husain S, Wagener MM, Singh N. Cryptococcus neoformans infection in organ transplant recipients: variables influencing clinical characteristics and outcome. Emerg Infect Dis 2001;7: 375-81.

5. Wu G, Vilchez RA, Eidelman B, Fung J, Kormos R, Kusne S. Cryptococcal meningitis: an analysis among 5,521 consecutive organ transplant recipients. Transpl Infect Dis 2002;4:183-8.

TO THE EDITOR: The case presentation notes that serologic testing showed the presence of antibodies against hepatitis B core and surface antigens; the test for hepatitis B surface antigen was negative. These results argue against the patient's having chronic hepatitis B disease. Fishman, in his discussion, states that the patient had end-stage liver disease due to hepatitis C and hepatitis B infections and alcoholism. With the positive tests for hepatitis $B$ antibodies and the negative test for surface antigen, it is not likely that hepatitis B contributed to the patient's liver disease. Fishman also states that reactivation of hepatitis B might occur in the course of treatment after transplantation. Although this is not impossible, the likelihood that hepatitis B would become reactivated seems remote, even in an immunosuppressed person, with the serologic findings noted in the patient under discussion. Furthermore, it also seems very unlikely that the patient would have been at risk for invasive fungal disease as a result of his prior hepatitis B infection.

Mark Joy, M.D., J.D.

Veterans Affairs New York Harbor Healthcare System Brooklyn, NY 11209

mark.joy@va.gov

THE DISCUSSANTS REPLY: Clinical suspicion is the key element in establishing a diagnosis of cryptococcal meningitis or for any infection of the central nervous system in immunocompromised hosts. All such patients with suspected central nervous system infection or persistent headache should undergo imaging of the central nervous system and, if appropriate, lumbar puncture with suitable diagnostic evaluations. ${ }^{1}$ As Antinori and Corbellino note, the serum or cerebrospinal fluid cryptococcal antigen assay is a useful assay for the diagnosis of infection in both immunocompromised hosts with AIDS and immunocompromised hosts without AIDS. If the suspicion of cryptococcal infection is high, a positive assay should suggest that the choice for initial therapy is an amphotericin product and that echinocandin therapy is likely to be ineffective. In addition, early attention to the possibility of increased intracranial pressures and combination therapy (with flucytosine) should be considered. In contrast, cryptococcal antigen is less useful in management. As in this patient, persistent elevation of cryptococ- 
cal antigen may occur even in patients without demonstrably viable organisms. The development of highly sensitive, specific, and quantitative diagnostic tools for fungal infections would improve the management of such life-threatening episodes.

In immunocompromised patients with fungal infections, various factors that may have contributed to the risk for infection need to be considered and, if possible, ameliorated. These factors include recent intensification of immunosuppression, concomitant viral infections, and unusual epidemiologic exposures. Thus, active infections (e.g., with cytomegalovirus) are treated to enhance the response to antifungal therapy. The ability to reduce the intensity of immunosuppression must be considered on an individual basis and carries some risk of graft rejection. Most recipients of solidorgan transplants have multiple risk factors; the contribution of individual factors is less certain. In patients with viral hepatitis, the relative con- tributions of active viral infection, cirrhosis, and the treatment of these infections are unclear. Thus, as Joy indicates, in the case under consideration, the contribution of hepatitis B is most likely to be through cirrhosis and for the risk of establishment of fungal colonization in the pretransplantation period and the potential risk of viral reactivation in the context of immunosuppression after transplantation. As the shortage of donor organs persists, the use of livers that are positive for core antigen, and thus for hepatitis B DNA, is likely to increase. The full effect of the use of donor organs according to expanded criteria has yet to be ascertained.

Jay A. Fishman, M.D.

Ramon Gilberto Gonzalez, M.D.

Massachusetts General Hospital

Boston, MA 02114

1. Fishman JA. Infection in solid-organ transplant recipients. N Engl J Med 2007;357:2601-14.

\section{Shifts in Health Information}

TO THE EDITOR: In their Sounding Board article on shifts in health information, Mandl and Kohane (April 17 issue) ${ }^{1}$ observe that "companies providing PCHRs [personally controlled health records] are not covered entities under the Health Insurance Portability and Accountability Act (HIPAA)," but incorrectly suggest that extending HIPAA to them would improve privacy. Publicly available PCHR systems are already prohibited from releasing information to private parties without the consent of the account holder under the federal Electronic Communications Privacy Act. ${ }^{2}$ However, HIPAA's privacy rule provision for disclosure without consent for "treatment, payment, or healthcare operations" (TPO) actually eliminates privacy. Although it at first appears to be quite reasonable, the decision about whether a specific disclosure is for TPO is made solely by the entity that holds the information, with no notice to the patient, no possibility for review or appeal, and no required audit trail. Clearly, the information holder is conflicted in making this decision and would tend to classify all desired disclosures as TPO. Further, the lack of audit-trail requirements for TPO disclosures means that there is no way to detect or assess abuse or violations. Therefore, extending HIPAA coverage would al- low the use of PCHR information without consent, nullifying consumer control and privacy.

William A. Yasnoff, M.D., Ph.D.

NHII Advisors

Arlington, VA 22201

william.yasnoff@nhiiadvisors.com

Deborah C. Peel, M.D.

Patient Privacy Rights Foundation

Austin, TX 78767

James C. Pyles, J.D.

Powers, Pyles, Sutter, and Verville, P.C.

Washington, DC 20005

1. Mandl KD, Kohane IS. Tectonic shifts in the health information economy. N Engl J Med 2008;358:1732-7.

2. 18 U.S.C. pt. I, ch. 121 , $\$ \S 2701-2712$ (1986). (Accessed June 20, 2008, at http://www.access.gpo.gov/uscode/title18/parti_ chapter121_html.)

TO THE EDITOR: Mandl and Kohane have tilted toward a more optimistic scenario for PCHRs than is currently warranted. Although they mention several potential problems, they have failed to mention one of the most serious issues: the potential undermining of the value, accuracy, and completeness of provider records. In this brave new world, every provider will still have the obligation to keep a clinical, legal, and business record of all patient encounters, and indeed, physi- 